\title{
Traduire
}

Ine autre perspective sur la tataduction

Revue française de la traduction

245 | 2021

Regards sur l'interprétation

\section{"Percer une obscurité cimmérienne ». Réflexions sur les techniques de traduction d'après Marie de Gournay}

Mykhailo Popovych

\section{(2) OpenEdition}

Journals

Édition électronique

URL : https://journals.openedition.org/traduire/2487

DOI : 10.4000/traduire.2487

ISSN : 2272-9992

Éditeur

Société française des traducteurs

Édition imprimée

Date de publication : 5 décembre 2021

Pagination : 99-106

ISSN : 0395-773X

Référence électronique

Mykhailo Popovych, « «Percer une obscurité cimmérienne ». Réflexions sur les techniques de traduction d'après Marie de Gournay », Traduire [En ligne], 245 | 2021, mis en ligne le 05 décembre 2021, consulté le 23 décembre 2021. URL : http://journals.openedition.org/traduire/2487 ; DOI : https://doi.org/10.4000/traduire.2487 


\section{"Percer une Obscurité cimmérienne»}

Réflexions sur les techniques de traduction d'après Marie de Gournay

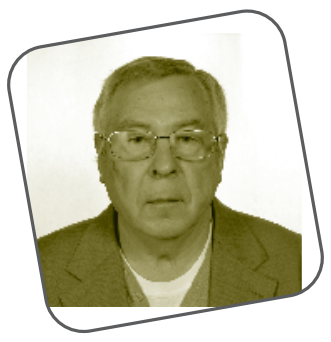

Mykhailo Popovych

Dans cette contribution, nous nous proposons de passer en revue les réflexions sur les techniques de traduction selon Marie de Gournay, telles qu'elle les exprime dans son traité Lettre à Monseigneur de Gelas, paru en 1626. Les réflexions de cette femme érudite, passionnantes à tout point de vue, sont peu connues, sinon oubliées, et donc loin d'être appréciées à leur juste valeur, en dépit des nombreuses recherches consacrées à ce sujet. Nous allons nous interroger sur ce qu'ont été les prémices de la traduction en France et sur l'apport fourni aux techniques de traduction par Marie de Gournay.

\section{Les prémices de la traduction en France}

Si l'on ne prend pas en compte les civilisations préromaines, pour lesquelles la traduction n'existe encore qu'à l'état embryonnaire, les premiers jalons de cette pratique ont été posés par Cicéron (106-43 av. J.-C.). Puis, ce fut au tour 
des traducteurs du Moyen Âge d'encourager sa progression, d'en faire un moyen donnant un accès direct à une autre culture et permettant aux érudits parlant différentes langues de s'entendre. Après mille ans de dépression médiévale, l'époque de la Renaissance donne une nouvelle impulsion au procédé consistant à se faire comprendre par un étranger en dépit de la diversité langagière et culturelle. Deux phénomènes favorisent cette évolution: 1) la substitution des langues vernaculaires au latin; 2) la soif des personnes instruites d'élargir leurs horizons à de nouvelles connaissances.

Dès la Renaissance, les traducteurs prennent en considération les acquis et les traditions séculaires de leurs nombreux devanciers et cherchent à édicter des règles de traduction permettant de reproduire toutes les qualités de l'original, tout en lui restant totalement fidèle. Cela a entraîné un tournant dans la traduction: considérée jusque-là comme un simple métier, elle devient progressivement un art et une science - et même "un art fondé sur une science», dira Georges Mounin quatre siècles plus tard (Mounin, 1964:13).

Ainsi, les premiers principes d'une nouvelle discipline, qui sera nommée "traductologie» $a u x x^{e}$ siècle, se sont nettement manifestés en Europe à l'époque de la Renaissance, surtout dans le champ intellectuel français

De l'avis de nombreux chercheurs, la Renaissance est une période propice à la traduction des ouvrages des penseurs antiques. Tout intellectuel connaissant une langue étrangère se croit capable, et même obligé, de traduire quelques textes, sans se rendre compte de ce qu'est en fait la traduction. Antoine Berman résume ainsi le développement des traductions à cette époque:

Donc tout le monde traduit, est pris par l'impulsion de traduire, de traduire de tout et n'importe quoi. Mais aussi, n'importe comment [...]. On s'attaque à des livres dont on ignore presque la langue et, surtout, on traduit sans aucun principe. (Berman, $1988: 25)$

Par conséquent, souligne-t-il en parfait accord avec Joachim du Bellay, "les mauvaises traductions abondent» (ibid.).

Bien entendu, un tel état de choses, du fait de la médiocre qualité des traductions, ne peut passer inaperçu dans les 
milieux cultivés, et ne peut perdurer. Avec la recrudescence des traductions au $x v^{e} I^{e}$ siècle apparaissent les premières réflexions sur la façon de traduire. L'honneur d'être le premier explorateur de ce «champ inculte» de la traduction revient à Étienne Dolet (1509-1546), écrivain, imprimeur et humaniste français. En 1540, il publie son célèbre traité La manière de bien traduire une langue en une autre dans lequel il établit cinq règles de bonne traduction, que tout traducteur devrait suivre.

«En premier lieu, il faut, écrit-il, que le traducteur entende parfaitement le sens et la matière de l'auteur qu'il traduit»(Dolet, 1540:13) ${ }^{1}$. Les quatre autres règles sont: avoir une parfaite connaissance des deux langues, ne pas traduire mot à mot, s'abstenir de mots hors de l'usage commun et respecter les règles de syntaxe, en évitant des «collocations impertinentes» (op. cit.: 18). Ces règles lui ont valu le titre de «père fondateur de la traductologie française».

Étienne Dolet a eu en France de nombreux successeurs qui se sont interrogés sur la nécessité d'établir des règles de traduction et ont commenté et complété celles qu'il a définies. Nous allons ainsi nous intéresser à l'héritage intellectuel de Marie le Jars de Gournay (1565-1645), femme d'une rare érudition pour son temps, philologue, écrivaine, traductrice et auteure d'innombrables traités dans lesquels elle dresse un panorama linguistico-culturel de la société française de son époque, pointant la diversité des problèmes qui dérangent.

\section{L'apport de Marie de Gournay à la technique de la traduction}

L'œuvre de Marie de Gournay est riche et variée. Dans une quarantaine de "discours de raison», recueillis dans Les Advis ou les Presens de la Demoiselle de Gournay (1641), elle traite de différents aspects de la vie de son époque, donnant son avis sur toutes sortes de questions posées par son temps.

Elle consacre plusieurs traités aux questions de traduction, notamment Lettre à Monseigneur de Gelas. De nombreuses

1. Nous avons, dans cette contribution, modernisé la graphie des citations des auteurs anciens pour une meilleure lisibilité et une meilleure compréhension. 
sources historiques attestent que Marie de Gournay a traduit du latin au français des ouvrages de plusieurs auteurs latins, sans compter des centaines de citations latines des Essais de Montaigne qu'elle a réédités plusieurs fois. De la sorte, sa propre conception de la traduction se présente comme une synthèse de son expérience personnelle de traductrice et de réflexions issues de sa pratique empirique.

La Lettre de Marie de Gournay à Claude Gelas, évêque d'Agen (1609-1630), peut être considérée comme une vraie introduction méthodologique aux œuvres qu'elle a traduites. On y lit notamment:

Si ceux qui liront les versions suivantes y voient quelque chose qui leur semble de prime abord hardiment traduite, ou brusquement, ils sont priés de se souvenir des précautions que j'ai proposées pour la traduction, en la lettre précédente, et de considérer ensuite que j'ai dû prendre pour moi les conseils que je donne aux autres pour ce regard. (Gournay, 1641: 641)

Dans cette lettre, l'essayiste réfléchit aux problèmes qui se posent au traducteur dans son travail, et affirme que

c'est une entreprise plus difficile et plus ardue qu'on ne peut croire que de tourner les grands auteurs, et les tourner en langue inférieure: surtout ornés d'un langage concis, magnifique, puissant et figuré, tel que celvi de Salluste ou de Tacite. (op. cit.: 635)

D'où, conclut-elle, le fait qu'il y a «tant de passables faiseurs de livres, et si peu de bons interprètes» (op. cit.: 637).

Partant de ce premier constat et se fondant sur sa propre pratique, Marie de Gournay procède à l'examen de diverses difficultés qui se présentent en cours de traduction. Elle montre que la nature de ces difficultés est variée et distingue notamment:

1) celles qui ont pour source l'état de la langue;

2) celles qui proviennent de l'œuvre à traduire;

3) celles qui ont pour origine l'intelligence et l'habileté du traducteur.

\section{L'état de la langue}

Cette mention ouvre la liste des problèmes que Marie de Gournay examine dans son traité. Dès le début de la 
Lettre, elle soulève de manière implicite le problème majeur de toute traduction, celui de la traduction fidèle. Est-il possible de "faire passer»ce qui est écrit du texte source au texte cible en cas de différences linguistiques entre les deux langues, surtout en cas d'«infériorité » de la langue cible? Elle ne donne pas de réponse clairement définie à cette question, se contentant de souligner à plusieurs reprises dans cette lettre, ainsi que dans d'autres écrits philologiques, que le français est inférieur au latin et qu'il faut l'enrichir par tous les moyens possibles afin qu'il soit capable de transmettre les plus fines nuances des ouvrages originaux. Et la traduction, répète-t-elle en écho au Cardinal du Perron, est "un effort très fructueux à l'enrichissement» de la langue française.

Marie de Gournay trouve que l'art d'exprimer "élégamment en un langage si différent de syntaxe» l'élégance des phrases des auteurs antiques, de l'exprimer en langage "davantage inférieur», demande de la part du traducteur «de belles inventions» (op. cit.: 636).

\section{Le caractère de l'œuvre à traduire}

Mademoiselle de Gournay note dans son traité que la qualité de la traduction peut dépendre du style de l'œuvre que l'on traduit, car il est des auteurs dont les écrits sont plus «faciles à traduire». Le traducteur doit alors choisir entre deux manières d'interpréter l'œuvre à traduire: soit l'exprimer "pertinemment, et non [en] coup de maître», soit «engendrer une œuvre de nouveau» (op. cit.: 636). Cette seconde option, dit la théoricienne, peut rendre l'œuvre traduite fort éloignée de la langue française. Or la tâche du traducteur consiste en la reproduction fidèle de l'œuvre originale, et non en la création d'une œuvre nouvelle. Dans ce cas, explique Marie de Gournay, les constructions de la langue que l'on traduit doivent être défaites "par une spéculation profonde et pénétrante, afin de les refaire par une autre pareille» (ibid.). Pourtant, un tel remaniement de la matière à traduire nécessite que le traducteur ait de l'esprit pour pouvoir la comprendre et la remodeler. Seulement, de tels esprits ne sont pas nombreux à l'époque de Marie de Gournay, qui regrette le manque "de bons interprètes» (op. cit.: 637). 


\section{L'intelligence et l'habileté du traducteur}

Si le thème de la langue et de ses qualités expressives est le sujet de prédilection des écrits philologiques de Marie de Gournay, elle se concentre par-dessus tout sur l'examen des compétences qu'un traducteur doit posséder pour pouvoir bien comprendre le contenu des textes qu'il traduit et pour que le lecteur retrouve dans la langue cible une translation fidèle.

Dans un premier temps, les pensées exposées par Marie de Gournay semblent faire écho aux règles de traduction établies par Étienne Dolet. Mais, en réalité, il n'en est rien. Les règles de Dolet ont un caractère purement empirique, tandis que les réflexions de notre auteure sont davantage théoriques. De plus, elles sont beaucoup plus clairvoyantes et profondes du point de vue scientifique et peuvent même être considérées comme les prémices de la future théorie de la traduction. Par exemple, Étienne Dolet enseigne, dans sa première règle, que tout traducteur doit "entendre parfaitement le sens de l'auteur, qu'il tourne d'une langue en une autre»car «sans cela, il ne peut traduire sûrement et fidèlement » (Dolet, 1540 :14). Marie de Gournay privilégie elle aussi le sens dans l'art de la traduction, mais son interprétation en est beaucoup plus profonde et analytique. "Pour dresser le sens et la phrase» de ce qu'il traduit, "le traducteur est obligé de détordre les mots à tous coups» et de se souvenir que ses connaissances grammaticales ne contribueront pas beaucoup à former "l'intelligence harmonique et mystique de ces écrits-là» (op. cit.: 637). Un bon traducteur, postule l'essayiste, doit savoir, pour recourir à une métaphore, "percer une obscurité cimmérienne», c'est-à-dire saisir l'énigme du sens contenu dans les formes verbales de ce qu'il traduit. Sans cela, il «ne fera jamais œuvre en ce métier» (ibid.).

La différence entre les deux auteurs est moins notable lorsqu'ils parlent de la compétence linguistique du traducteur. Selon Étienne Dolet, le traducteur doit avoir une "parfaite connaissance de la langue de l'auteur qu'il traduit» et être "excellent en la langue en laquelle il se met à traduire» (Dolet, $1540: 15$ ). Marie de Gournay exprime une idée similaire, mais précise dans le même temps que le traducteur qui s'attelle aux ouvrages d'auteurs anciens doit parler le français «non seulement aussi nettement, mais aussi vigoureusement, 
richement, figurément, succinctement, et délicieusement, qu'ils parlent [leur langue]». En outre, il doit être «savant en français plus qu'il ne l'est en latin» (op. cit.: 637).

Pour faire une bonne traduction, poursuit-elle, il ne suffit pas de maîtriser parfaitement deux langues, il faut aussi être capable de comprendre la conception de l'auteur dont on traduit l'œuvre, et de pouvoir "l'expliquer en bons termes» (op. cit.: 636). Et cela ne peut se faire sans une bonne connaissance de l'auteur traduit, et sans l'intervention de l'intelligence du traducteur. Suivant sa vision, pour faire une traduction de qualité,

il est nécessaire de ruminer, considérer, juger, approfondir et savoir à fer émoulu: je dis savoir, l'auteur qu'on entreprend et mille autres, pour se démêler des difficultés par confrontations et rapports. (op. cit.: 637)

En conclusion, on peut affirmer que, dans la conception de Marie de Gournay, traduire n'est pas un acte irréfléchi, machinal, un «labeur de pure peine et diligence: labeur [...] de transporter une chose de place en autre, sans invention» (op. cit.: 636). Elle a démontré que traduire une œuvre est un processus extrêmement complexe, qui implique de la part du traducteur qu'il accomplisse de nombreux efforts pour recréer au moyen de sa propre langue tout un univers d'abord créé dans une langue étrangère, un univers composé de faits linguistiques et extralinguistiques. Le traducteur doit parfaitement connaître les deux systèmes linguistiques avec lesquels il travaille, comprendre la culture dans laquelle s'est formée la conception philosophique de l'écrivain traduit, prendre en «considération des temps, lieux et sujets» (op. cit.: 639) et bien d'autres phénomènes sans lesquels l'œuvre traduite ne pourrait prétendre à «un entendement frère cadet » de l'original (ibid.).

En résumé, on peut dire que Marie de Gournay a toute sa place dans les débats contemporains sur la traduction et que ses idées sur l'art de la traduction sont toujours stimulantes, en dépit des cinq siècles qui nous séparent d'elle. 
Mykhailo POPOVyCH est enseignant-chercheur au département de philologie romane et de traduction de l'Université nationale Yuriy Fedkovych de Tchernivtsi en Ukraine. II a soutenu en 1982 une thèse de docteur ès lettres portant sur les répétions nominales dans le texte. De 1995 à 1999, il a été lecteur de langue ukrainienne à l'Université Paris 8 . Profitant des ressources scientifiques des bibliothèques parisiennes, il a préparé une thèse de docteur d'État (HDR) intitulée La détermination/ l'indétermination du nom dans le discours, soutenue en 2002. Auteur de plusieurs manuels et de nombreux articles portant sur diverses questions relatives à la langue française, il a été fait chevalier dans l'ordre des Palmes académiques (2015).

SOURCES CITÉES

BERMAN Antoine, «De la translation à la traduction », in TTR: traduction, terminologie, rédaction, I(1), 1988, p. 23-40, https://doi.org/10.7202/037002ar

DOLET Estienne, La manière de bien traduire d'une langue en autre, Lyon, 1540, https:/gallica.bnf.fr/ark:/12148/bpt6k505680.image, consulté le 09/08/2021.

GOURNAY Marie, Les Advis, ou, les Presens de la Demoiselle de Gournay, zè éd. augmentée, revue et corrigée, Paris, J. Du Bray, 1641, https:/gallica.bnf.fr/ark:/12148/ bpt6k71929v.image, consulté le 09/08/2021.

MOUNIN Georges, Les problèmes théoriques de la traduction, Paris, Éditions Gallimard, 1963. 